3.Hehir, K.M., D. Armentano, L.M. Cardoza, T.L. Choquette, P.B. Berthelette, G.A. White, L.A. Couture, M.B. Everton, et al. 1996. Molecular characterization of replication-competent variants of adenovirus vectors and genome modifications to prevent their occurrence. J. Virol. 70:8459-8467.

4.Fallaux, F.J., A. Bout, I. van de Velde, D.J.M. van den Wollenberg, K. Hehir, J. Keegan, C. Auger, S. Cramer, et al.1998. New helper cells and matched early region 1-deleted adenovirus vectors prevent generation of replication-competent adenoviruses. Hum. Gene Ther. 9:1909-1917

5.Pau, M.G., C. Ophorst, M.H. Koldijk, G. Schouten, M. Mehtali, and F. Uytdehaag. 2001. The human cell line PER.C6 provides a new manufacturing system for production of influenza vaccines. Vaccine 19:2716-2721.

6.Jones, D., N. Kroos, R. Anema, B. van Montfort, A. Vooys, S. van der Kraats, E. van der Helm, S. Smits, et al. 2003. High-level expression of recombinant $\operatorname{IgG}$ in the human cell line PER.C6. Biotechnol. Prog. 19:163-168.

7.Szuhai, K., V. Bezrookove, J. Wiegant, J. Vrolijk, R.W. Dirks, C. Rosenberg, A.K. Raap, and H.J. Tanke. 2000. Simultaneous molecular karyotyping and mapping of viral DNA integration sites by 25-color COBRAFISH. Genes Chromosomes Cancer 28:92-97.

8.Wiegant, J. and A.K. Raap. 1998. Basic preparative techniques for fluorescence in situ hybridization, 8.3.1-8.3.21. In J.P. Robinson (Ed.), Current Protocols in Cytometry. John Wiley \& Sons, New York.

9.Tanke, H.J., J. Wiegant, R.P.M. van Gijlswijk, V. Bezrookove, H. Pattenier, R.J. Heetebrij, E.G. Talman, A.K. Raap, et al. 1999. New strategy for multi-colour fluorescence in situ hybridization: COBRA: combined binary ratio labelling. Eur. J. Hum. Genet. 7:2-11.

10.Nichols, W.W., R. Lardenoije, B.J. Ledwith, K. Brouwer, S. Manam, R. Vogels, D. Kaslow, D. Zuidgeest, et al. 2002. Propagation of adenoviral vectors: use of PER.C6 cells, p. 129-166. In D. Curiel and J. Douglas (Eds.), Adenoviral Vectors for Gene Therapy. Elsevier Science, New York.

11.Rabbits, P., H. Impey, A. Heppell-Parton, C. Langford, C. Tease, N. Lowe, D. Bailey. M. Ferguson-Smith, and N. Carter. 1995. Chromosome specific paints from a high-resolution flow karyotype of the mouse. Nat. Genet. 9:369-375.

12.Balajee, A.S., I. Dominguez, and A.T. Natarajan. 1995. Construction of Chinese hamster chromosome specific DNA libraries and their use in the analysis of spontaneous chromosome rearrangements in different cell lines. Cytogenet. Cell Genet. 70:95-101.

Received 9 March 2004; accepted 26 April 2004.

Address correspondence to Joop Wiegant, Leiden University Medical Center, Department of Molecular Cell Biology, Wassenaarseweg 72, 2333 AL Leiden, The Netherlands.e-mail:J.C.A.G.Wiegant@lumc.nl

\title{
Identifying adaptor contamination when mining DNA sequence data
}

\author{
Jeffrey Scott Coker and Eric Davies \\ North Carolina State University, Raleigh, NC, USA
}

BioTechniques 37:194-198 (August 2004)

Analysis of DNA sequences can only be as correct as the sequences themselves, and so contamination in public databases is a major concern for bioinformatics research. Here we describe a simple screen that identified adaptor contamination in over 78,000 eukaryotic sequences in GenBank ${ }^{\circledR}$. Awareness that over $99 \%$ of adaptor contaminants appear near the ends of sequences, are flanked by a vector, or involve adaptor dimerization allows the detection of 99\% of these sequences (Figure 1).

A contaminated sequence is defined as "one that does not faithfully represent the genetic information from the biological source organism/organelle because it contains one or more sequence segments of foreign origin" (http://www. ncbi.nlm.nih.gov/VecScreen/contam. html). Sources of contamination for nuclear DNA and cDNA include vector sequence (1-6), plasmid vector insertion sequences (7), impure tissue sources (8), faulty laboratory protocols $(9,10)$, mitochondrial DNA (11), and ribosomal DNA/RNA (12). There is one published account of contamination due to adaptor sequences, where it was shown that commercial adaptor sequences matched the $5^{\prime}$ or $3^{\prime}$ end of 728 GenBank and the European Molecular Biology Laboratory (EMBL) sequences (13). Strategies to decrease contamination in database sequences have emphasized vector sequences $(4-6,8)$ and given little attention to adaptor contamination.

An adaptor is a short oligonucleotide that is ligated to the ends of cDNAs for incorporation into a vector cloning site (Figure 1). Usually adaptors consist of several restriction sites, one blunt end (for ligation to cDNA) and one cohesive end (for ligation to a vector). Adaptors are frequently used in the construction of cDNA libraries and in generating cDNA ends using rapid amplification of cDNA ends (RACE) PCR.

The presence of adaptor sequences in organismal sequences in public databases has the potential to cause many different errors of interpretation $(14,15)$, which include the following: (i) false hits for others using public databases; (ii) added difficulties in identifying genes and joining contigs; (iii) misconstruction of PCR primers, microarrays, probes, etc.; (iv) incorrect conclusions regarding evolution and differences between organisms; (v) incorrect conclusions about gene structure, mRNA splicing, and mRNA transport; and (vi) incorrect conclusions about protein sequence, structure, transport, and function.

To investigate adaptor contamination in public databases, BLASTn searches of GenBank (release 140.0; Feb. 15, 2004) eukaryotic sequences were performed using the search parameters shown in Table 1 . The search parameters returned perfect matches (100\% identity) with the respective adaptor sequences (Table 1). It should be noted that three separate searches of the expressed sequence tag (EST) databases were performed for ZAP (Stratagene, La Jolla, CA, USA) and P1/PN1 (BD Biosciences Clontech, San Jose, CA, USA) adaptors (human, mouse, and non-human/mouse ESTs were searched separately using the E-values in Table 1) because searching all ESTs simultaneously returned more hits than the server could process. Manual review of individual GenBank entries, literature review, and personal communications were used to investigate several hundred matches further. GenBank entries with adaptor contamination were also screened for vector contamination using VecScreen (www.ncbi.nlm.nih.gov/VecScreen/ VecScreen.html), the tool commonly 
used to screen GenBank submissions.

The searches and subsequent analyses identified over 78,000 contaminated sequences in GenBank (Table 1). Most contaminated sequences were found in the GenBank EST database, but the "nr" database (which contains annotated genes, etc.) also contained 4528 false sequences. There were also a large number of shorter matches with adaptors that were not included when using the search parameters in Table 1, making it evident that the actual number of contaminated sequences is much higher than shown in Table 1. Simply increasing the E-value will return these shorter matches.
Within the contaminated GenBank sequences, over $99 \%$ of adaptors were within $50 \mathrm{bp}$ of an end, connected to vector sequence match as shown by VecScreen, or involved in dimerization (Figure 1). The majority of matches not near the $5^{\prime}$ or $3^{\prime}$ end involved dimerization of the ZAP adaptor as shown in Figure 1. We performed BLASTn searches using the full sequences of many GenBank entries that included putative dimer sequences in the gene or cDNA sequence. These searches typically resulted in some GenBank entries matching the query on one side of the dimer, but had totally different entries matching the

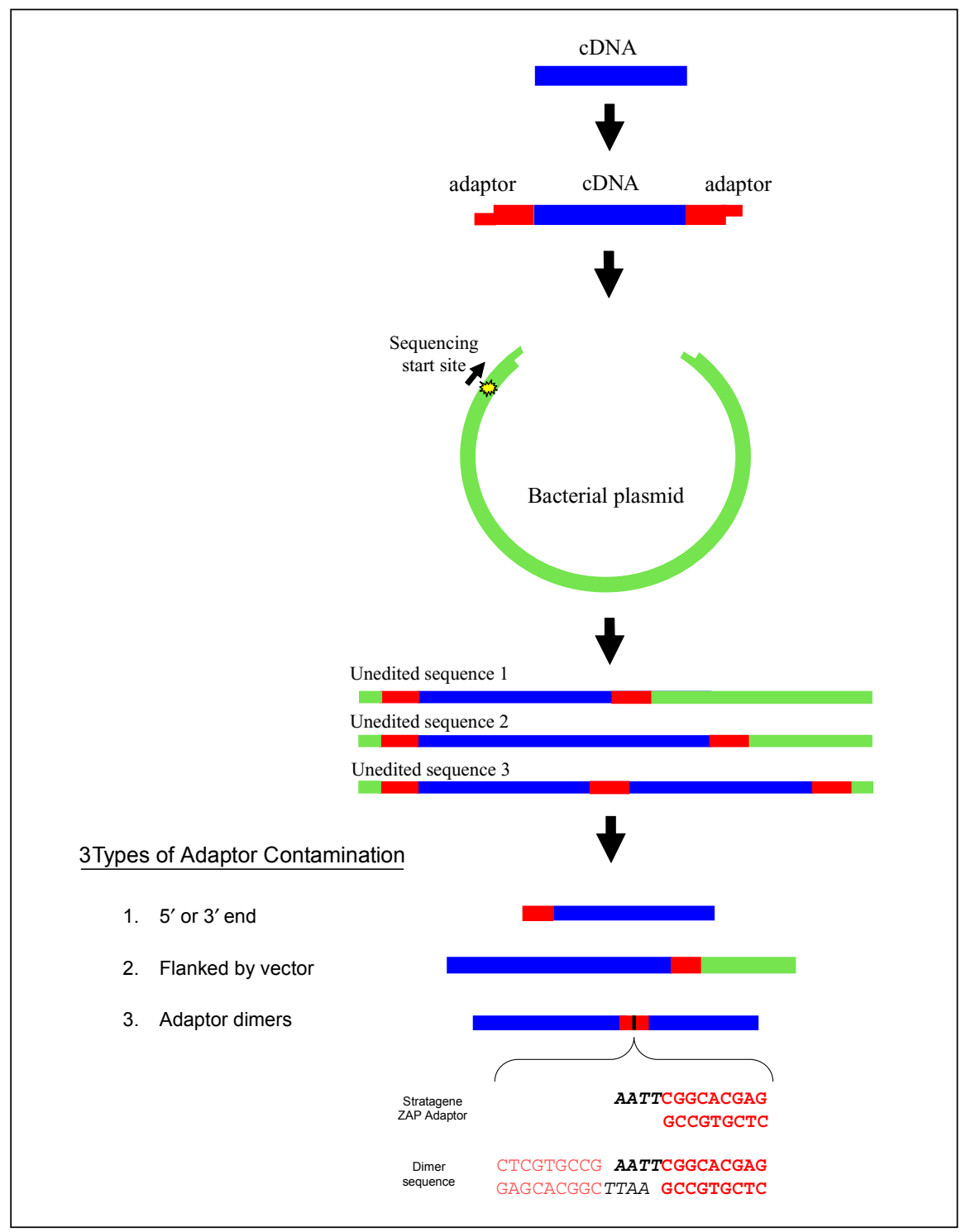

Figure 1. The path from sequencing a cDNA to an improperly edited sequence. More than $99 \%$ of sequences contaminated with adaptors fall into one of the three groups shown at the bottom. other side, suggesting that the query sequences actually contained two unrelated sequences that were joined via dimerization. Obviously, this has the potential to create significant errors, especially since the dimer is often in the middle of sequences where it is more likely to be interpreted as part of the open reading frame.

A subset of 210 matches (from the $\mathrm{nr}$ database) with BD Biosciences Clontech's Marathon primer adaptors (Table 1) were examined more closely. These adaptors are part of the company's suppression subtractive hybridization procedure (U.S. patents 5,565,340 and $5,759,822$ ) used originally to make cDNA libraries and probes $(16,17)$. Of 210 matches with the Marathon adaptors, at least $82(39 \%)$ are contaminated in regions designated as gene or cDNA sequence, including 11 open reading frames $(5 \%)$. Through literature review and personal communications, we confirmed that $\mathrm{BD}$ Bioscience Clontech protocols had been used. Published literature shows these false sequences appearing in transposons, protein sequences, regions used to join contigs, and other biologically relevant regions. In fact, we found published accounts of (unrecognized) contaminated sequence in most major journals of genetics and molecular biology.

Public sequence databases such as GenBank serve as repositories for primary sequence data, and typically sequences are not altered or removed without a submitter's request. Thus, dealing with adapter contamination is more complex than simply removing the false sequences from public databases. Future efforts to reduce adaptor contamination in public databases should include: $(i)$ a discussion of sequence quality control in cDNA library product literature; (ii) greater diligence by researchers; (iii) improved tools for screening database submissions; (iv) checks for adaptor contamination during sequence curation (i.e., GenBank RefSeqs); ( $v$ ) better communication between those who find mistakes, those who maintenance the databases, and sequence submitters; and (vi) prompt correction by submitters who realize past mistakes.

For those mining data, performing searches similar to those in Table 1 
Table 1. Sequences and Search Parameters to Identify Entries in GenBank Contaminated by Seven Commercial Adaptor Sequences

\begin{tabular}{|c|c|c|c|c|c|c|c|c|}
\hline \multirow[b]{2}{*}{ Adaptor } & \multirow[b]{2}{*}{ Sequence to Search } & \multirow{2}{*}{$\begin{array}{l}\text { Detected by } \\
\text { VecScreen? }\end{array}$} & \multicolumn{4}{|c|}{ Search Parameters } & \multicolumn{2}{|c|}{ Matches in Eukaryota } \\
\hline & & & Filter & E-Value & Word Size & Identity & nr Database & EST Database \\
\hline $\begin{array}{l}\text { BD Biosciences } \\
\text { Clontech P1/PN1 }\end{array}$ & 5'-TCGAGCGGCCGCCCGGGCAGGT-3' & $3^{\prime} \quad$ Yes & none & 1.00 & 7 & 100 & 255 & 11,655 \\
\hline $\begin{array}{l}\text { BD Biosciences } \\
\text { Clontech P2/PN2 }\end{array}$ & 5'-AGGGCGTGGTGCGGAGGGCGGT-3' & $3^{\prime} \quad$ No & none & 1.00 & 7 & 100 & 13 & 705 \\
\hline $\begin{array}{l}\text { BD Biosciences } \\
\text { Clontech EcoRI }\end{array}$ & 5'-AATTCGCGGCCGCGTCGAC-3' & Yes & none & 0.05 & 7 & 100 & 156 & 15,071 \\
\hline Stratagene ZAP & 5'-AATTCGGCACGAG-3' & No & none & 150.0 & 7 & 100 & 3166 & 28,830 \\
\hline $\begin{array}{l}\text { Stratagene ZAP } \\
\text { (dimer) }\end{array}$ & 5'-CTCGTGCCGAATTCGGCACGAG-3' & No & none & 0.005 & 7 & 100 & (778) & $(24,106)$ \\
\hline Life Technologies & 5'-GGCCACGCGTCGACTAGTAC-3' & Yes & none & 10.0 & 7 & 100 & 53 & 66 \\
\hline
\end{tabular}

(varying parameters as appropriate to return $100 \%$ matches with the appropriate adaptors) will uncover possible cases of adaptor contamination. Matches that fall into one of the three classes shown in Figure 1 are almost certainly false sequences. When using many sequences from the same library, it is best to determine what cloning technology was used and search for the appropriate adaptors. If one does not know specific adaptors, another technique is to BLAST the first and/or last $30 \mathrm{bp}$ of all sequences against one another and look for sequences that are overrepresented. Doubts about whether a sequence is an adaptor contaminant can be resolved by finding sequences posted by other laboratories or determining what technology was used to clone and sequence the cDNA.

The recognition of adaptor contamination has the potential to resolve many problems in the literature $(14,15)$. It is expected that removing adaptor contamination will clarify many gene sequences as individual laboratories reinterpret their own sequences and will prevent those mining data from amplifying such errors.

\section{ACKNOWLEDGMENTS}

We thank the scientists who corresponded with us regarding their GenBank entries, Sophia Clotho for advice, Ron Sederoff for critical review, and staff at National Center for Biotechnology Information (NCBI) for their correspondence.

\section{COMPETING INTERESTS STATEMENT}

The authors declare that they have no competing interests.

\section{REFERENCES}

1.Lamperti, E.D., J.M. Kittelberger, T.F. Smith, and L. Villakomaroff. 1992. Corruption of genomic databases with anomalous sequence. Nucleic Acids Res. 20:2741-2747.

2.Lopez, R., T. Kristensen, and H. Prydz. 1992. Database contamination. Nature 355:211.

3.Reynolds, T.L. 1994. Vector DNA artifacts in the nucleotide-sequence database. BioTechniques 16:1124-1125.

4.Harger, C., M. Skupski, J. Bingham, A. Farmer, S. Hoisie, P. Hraber, D. Kiphart, L. Krakowski, et al. 1998. The Genome Sequence DataBase (GSDB): improving data quality and data access. Nucleic Acids Res. 26:21-26.

5.Miller, C., J. Gurd, and A. Brass. 1999. A RAPID algorithm for sequence database comparison: application to the identification of vector contamination in the EMBL databases. Bioinformatics 15:111-121.

6.Seluja, G.A., A. Farmer, M. McLeod, C. Harger, and P.A. Schad. 1999. Establishing a method of vector contamination identification in database sequences. Bioinformatics 15:106-110.

7.Binns, M. 1993. Contamination of DNA database sequence entries with Escherichia coli insertion sequences. Nucleic Acids Res. 21:779-779.

8.White, O., T. Dunning, G. Sutton, M. Adams, J.C. Venter, and C. Fields. 1993. A quality-control algorithm for DNA-sequencing projects. Nucleic Acids Res. 21:3829-3838.

9.Gersuk, V.H. and T.M. Rose. 1993. Database contamination. Science 260:606.

10.Dean, M. and R. Allikmets. 1995. Contamination of cDNA libraries and expressed- sequence-tags databases. Am. J. Hum. Genet. 57:1254-1255.

11.Wenger, R.H. and M. Gassmann. 1995. Mitochondria contaminate databases. Trends Genet. 11:167-168.

12.Gonzalez, I.L. and J.E. Sylvester. 1997. Incognito rRNA and rDNA in databases and libraries. Genome Res. 7:65-70.

13.Yoshikawa, T., A.R. Sanders, and S.D. Detera Wadleigh. 1997. Contamination of sequence databases with adaptor sequences. Am. J. Hum. Genet. 60:463-466.

14.Coker, J.S. and E. Davies. 2002. Correspondence re: A.H. Ree, et al., Expression of a Novel Factor in Human Breast Cancer Cells with Metastatic Potential (Cancer Res., 59: 4675-4680, 1999). Cancer Res. 62:4164-4165.

15.Forster, P. 2003. To err is human. Ann. Hum. Genet. 67:2-4.

16.Diatchenko, L., Y.-F. Chris Lau, A.P. Campbell, A. Chenchik, F. Moqadam, B. Huang, S. Lukyanov, K. Lukyanov, et al. 1996. Suppression subtractive hybridization: a method for generating differentially regulated or tissue-specific cDNA probes and libraries. Proc. Natl. Acad. Sci. USA 93:6025-6030.

17.Jin, H., X. Cheng, L. Diatchenko, P.D. Siebert, and C.C. Huang. 1997. Differential screening of a subtracted cDNA library: a method to search for genes preferentially expressed in multiple tissues. BioTechniques 23:1084-1086.

Received 8 April 2004; accepted 10 May 2004.

Address correspondence to Jeffrey Scott Coker, Elon University, Department of Biology, 2625 Campus Box, Elon, NC, 27244, USA.e-mail: jeffreycoker@hotmail.com 